\title{
When Rotational Atherectomy is not Enough
}

\author{
Negar Atefi ${ }^{1}$, Basem Elbarouni ${ }^{2}$, Amir Ravandi $^{2}$, and David Allen ${ }^{2}$ \\ ${ }^{1}$ University of Manitoba \\ ${ }^{2}$ University of Manitoba College of Medicine
}

November 27, 2020

\begin{abstract}
Severe coronary calcification predicts stent under-expansion, malapposition, and poor outcomes in percutaneous coronary intervention. Rotational atherectomy permits debulking of coronary calcium allowing for stent expansion. More recently, intravascular lithotripsy has been used to fracture calcium and aid revascularization. Here we report two cases where both strategies were required for revascularization.

Title - When Rotational Atherectomy is not Enough

Short Title - Extreme Coronary Calcification

Negar Atefi ${ }^{1}$, Basem Elbarouni ${ }^{1,2}$, Amir Ravandi $^{1,2}$, David W Allen ${ }^{1,2}$

${ }^{1}$ Max Rady College of Medicine, University of Manitoba 727 McDermot Avenue, Winnipeg MB, CAN R3E $3 \mathrm{P} 5$

${ }^{2}$ St Boniface General Hospital, Section of Cardiology 409 Tache Avenue, Winnipeg, MB, CAN R2H 2A6

Corresponding Author:

David W Allen MD FRCPC

Assistant Professor

Section of Cardiology,

Max Rady College of Medicine, University of Manitoba, Canada

409 Tache Avenue, RmY3543

Winnipeg, Manitoba, Canada

R2H $2 \mathrm{~A} 6$
\end{abstract}

Ph 204-237-2390 Fax 204-233-4853

dallen@sbgh.mb.ca

Conflicts: None

Word Count 1505 (including references)

Key words: Cardiovascular disorders 
Key Clinical Message: Extreme coronary calcification may require rotational atherectomy to create a navigable intravascular lumen followed by intravascular lithotripsy to fracture areas of deep calcification to allow for successful percutaneous coronary intervention.

Abstract

Severe coronary calcification predicts stent under-expansion, malapposition, and poor outcomes in percutaneous coronary intervention. Rotational atherectomy permits debulking of coronary calcium allowing for stent expansion. More recently, intravascular lithotripsy has been used to fracture calcium and aid revascularization. Here we report two cases where both strategies were required for revascularization.

Introduction

Calcified coronary lesions are more likely to result in stent malapposition and expansion resulting in higher rates of restenosis, thrombosis, and myocardial infarction (1). In this patient population, fastidious lesion preparation is critical to ensure that calcium fracture is sufficient to allow for stent expansion. Commonly used tools include non-compliant (NC) balloons, cutting balloons, scoring balloons and atherectomy. Recently, intravascular lithotripsy (IVL) has been used for recalcitrant lesions and may supplant atherectomy owing to its ease of use, risk profile and long-term risk profile (2). Atherectomy on the other hand, is best reserved for specialized, high-volume centers. However, even in these settings, atherectomy is a time consuming and costly procedure associated with an increased risk of procedural complications $(2,3)$. However, IVL is only effective if it can be delivered to the site of coronary calcification. Thus, atherectomy and IVL may not be mutually exclusive techniques, some lesions require atherectomy to gain intimal passage for IVL to fracture deeper layers of calcium for successful revascularization $(4,5)$.

Case 1:

A 68-year-old male with a history of coronary artery disease with previous PCI to the right coronary artery, diabetes mellitus, hypertension, dyslipidemia, and gout presented with chest pain and was diagnosed with a non-ST elevation myocardial infarction (NSTEMI). Angiogram identified a severe mid-LAD calcified lesion (Figure 1.A). Balloon angioplasty was performed with compliant and $\mathrm{NC}$ balloons with failure to yield (Figure 1.B). Therefore, the procedure was stopped, and the patient returned for dedicated atherectomy. A 7 French radial system was used to perform rotational atherectomy with a $1.75 \mathrm{~mm}$ and a $2.0 \mathrm{~mm}$ burr. Despite this, the lesion would not yield in spite of high-pressure balloon inflation after each run (Figure 1.C and 2.E). Optical Coherence Tomography (OCT) was performed after each attempt and confirmed the presence of a $1.75 \mathrm{~mm}$ and $2.0 \mathrm{~mm}$ lumen surrounded by dense circumferential calcification (Figure 1.D and 2.F). Therefore, intravascular lithotripsy (Shockwave Medical, Santa Clara, CA, USA) was performed. A 3.5mm shockwave IVL balloon was delivered and 8 rounds of lithotripsy were performed. Subsequent OCT confirmed multiple fractures within the lesion (Figure 1.G). A Promus Premier (Boston Scientific, Marlborough, MA, USA) $3.5 \times 32 \mathrm{~mm}$ drug-eluting stent was delivered and post-dilated using a $4.0 \times 20 \mathrm{~mm}$ NC balloon proximally and a $3.75 \mathrm{~mm} \mathrm{NC}$ balloon distally with excellent results (Figure $1 . \mathrm{H}$ ).

Case 2:

A 79-year-old male with a prior history of PCI to the proximal LAD presented with NSTEMI and was referred for coronary angiography. The culprit lesion was proximal LAD with total occlusive in-stent restenosis (ISR) (Figure 2.A). Successful antegrade wiring of the occlusion was performed. Despite multiple NC balloons and cutting balloons, the lesion remained resistant to expansion (Figure 2.B). Intravascular ultrasound (IVUS) confirmed highly calcified lesion within the previous stent. Atherectomy was performed using a $1.5 \mathrm{~mm}$ and a $2.0 \mathrm{~mm}$ burr. However, the lesion was resistant to expansion with $\mathrm{NC}$ and cutting balloons (Figure 2.C and 2.D). A distal dissection developed and was stented with a $2.5 \times 28 \mathrm{~mm}$ Promus Premier drug-eluting stent (Figure 2.E). At this point, the procedure was stopped and the patient brought back for IVL. IVL was performed using a $3.5 \mathrm{~mm}$ Shockwave balloon for 6 cycles. This allowed for successful pre-dilatation of the calcified lesion with a $4.0 \mathrm{~mm} \mathrm{NC}$ balloon at high pressure. This allowed the placement of a $4.0 \mathrm{x} 24 \mathrm{~mm}$ Promus Premier drug-eluting stent to be placed with excellent results (Figure 2.G). 


\section{Discussion}

The cases discussed above demonstrate that even large burr rotational atherectomy in heavily calcified lesions may not sufficiently prepare the lesion for expansion. In both cases multiple rounds of atherectomy were attempted after the failure of traditional lesion preparation techniques. IVL was not initially attempted as the imaging and introduction of smaller devices demonstrated that the IVL balloon could not be delivered to the lesion. Atherectomy was required to develop an accessible lumen, but was not sufficient to fracture the dense circumferential calcification. In both cases, intravascular imaging was critical to identification of the true vessel size and the burden of calcification resulting in use of IVL to fracture the residual lesion. The Disrupt CAD I and II studies have demonstrated that IVL is particularly suited for lesions with circumferential calcification; it results in multiple planes of fracture and is not prone to wire bias, a known limitation of atherectomy $(3,6)$. These studies also demonstrated a favorable procedural complication rate and good longer-term outcomes. Therefore, IVL and rotational atherectomy can be complementary to one another and may occasionally be used in combination to provide better results in patients with severely calcific lesions (7). Rotablation ablates calcium in the intima and allows for an accessible passage for balloons and stents, IVL utilizes this passage and treats the circumferential deep calcium layers of the lesion. Proficiency in both techniques is required when handling lesions of this subset.

Conclusion

Extreme calcification may require atherectomy and IVL to achieve optimal revascularization results and interventional cardiologists should be familiar with the use and limitations of each technique.

Acknowledgements

None.

Conflict of Interest

No funding or conflict of interest to disclose for Mrs Atefi, Dr's Elbarouni, Ravandi or Allen.

Author contributions

NA wrote the manuscript, DWA \& BE contributed the presented cases. AR and DWA contributed to the design and critically reviewed and revised the manuscript.

\section{References}

1. Danek BA, Brilakis ES. Serenity, courage, and wisdom: the keys to successful coronarycalcification treatment. Catheter Cardiovasc Interv. 2016;88:897-898.

2. Venuti G, D'Agosta G, Tamburino C, La MannaA. Coronary lithotripsy for failed rotational atherectomy, cutting balloon, scoring balloon, and ultra-high-pressure non-compliant balloon. Catheter Cardiovasc Interv. 2019;94:111-115.

3. Ali ZA, Nef H, Escaned J, Werner N, Banning AP, Hill JM, De Bruyne B, Montorfano M, Lefevre T, Stone GW, Crowley A, Matsumura M, Maehara A, Lansky AJ, Fajadet J, Di Mario C. Safety and Effectiveness of Coronary Intravascular Lithotripsy for Treatment of Severely Calcified Coronary Stenoses: The Disrupt CAD II Study. Circ Cardiovasc Interv. 2019;12(10):e008434. doi: 10.1161/CIRCINTERVENTIONS.119.008434. Epub 2019 Sep 25. PMID: 31553205.

4. Ali ZA, Brinton TJ, Hill JM, Maehara A, Matsumura M, Karimi Galougahi K, Illindala U, Götberg M, Whitbourn R, Van Mieghem N, Meredith IT, Di Mario C, Fajadet J. Optical Coherence Tomography Characterization of Coronary Lithoplasty for Treatment of Calcified Lesions: First Description. JACC Cardiovasc Imaging. 2017 Aug;10(8):897-906. doi: 10.1016/j.jcmg.2017.05.012. PMID: 28797412.

5. Nagaraja V, Ubaid S, Khoo C, Ratib, K. Intravascular lithotripsy for stent under-expansion despite utilization of rotational atherectomy for plaque modification. Cardiovasc Revasc Med 2019;17:S15538389(19)30677-3. doi: 10.1016/j.carrev.2019.10.024. PMID 31862170.

6. Brinton TJ, Ali ZA, Hill JM, Meredith IT, Maehara A, Illindala U, Lansky A, Götberg M, Van Mieghem NM, Whitbourn R, Fajadet J, Di Mario C. Feasibility of shockwave coronary intravascu- 
lar lithotripsy for the treatment of calcified coronary stenoses. Circulation. 2019;139(6):834-836. doi: 10.1161/CIRCULATIONAHA.118.036531. PMID: 30715944.

7. Jurado-Román A, Gonzálvez A, Galeote G, Jiménez-Valero S, Moreno R. RotaTripsy: Combination of Rotational Atherectomy and Intravascular Lithotripsy for the Treatment of Severely Calcified Lesions. JACC Cardiovasc Interv. 2019;12(15):e127-e129. doi: 10.1016/j.jcin.2019.03.036. Epub 2019 Jul 17. PMID: 31326422.

Figure 1. (A) Pre-procedure angiogram of mLAD lesion(arrow) in anterior-posterior (AP) cranial view. (B)Failure to expand with a $3.5 \mathrm{~mm} \mathrm{NC}$ balloon to high pressure(arrows) . (C) Failure to expand with a $3.5 \mathrm{~mm} \mathrm{NC}$ balloon after rotational atherectomy with a $1.75 \mathrm{~mm}$ burr(arrows) (D) OCT post $1.75 \mathrm{~mm}$ bur rotational atherectomy confirming formation of a $1.73 \mathrm{~mm}$ diameter lumen.(E) Failure to expand with a $3.5 \mathrm{~mm} \mathrm{NC}$ balloon to high pressure (arrow) after $2.0 \mathrm{~mm}$ burr rotational atherectomy and (F) Repeat OCT confirming $2.0 \mathrm{~mm}$ lumen with dense calcification (G) OCT image post-IVL demonstrating fractures in the lesion. (H)Post-PCI angiogram showing successful revascularization in mLAD

(arrow).

Figure 2. (A) Primary angiogram of pLAD showing ISR(arrow) in the right anterior oblique (RAO) cranial view.(B) Failure to expand the lesion with $2.5 \mathrm{~mm}$, and $3.5 \mathrm{~mm}$ cutting and NC balloons (arrows). Failed atherectomy with (C)1.5mm burr and (D) $2.0 \mathrm{~mm}$ burr (arrows). (E) Angiogram post PCI of mLAD dissection revealing persistent pLAD lesion(F) Post-IVL IVUS showing fractures in the lesion(arrows) and (G) final angiogram after PCI showing successful revascularization of pLAD (arrow). 


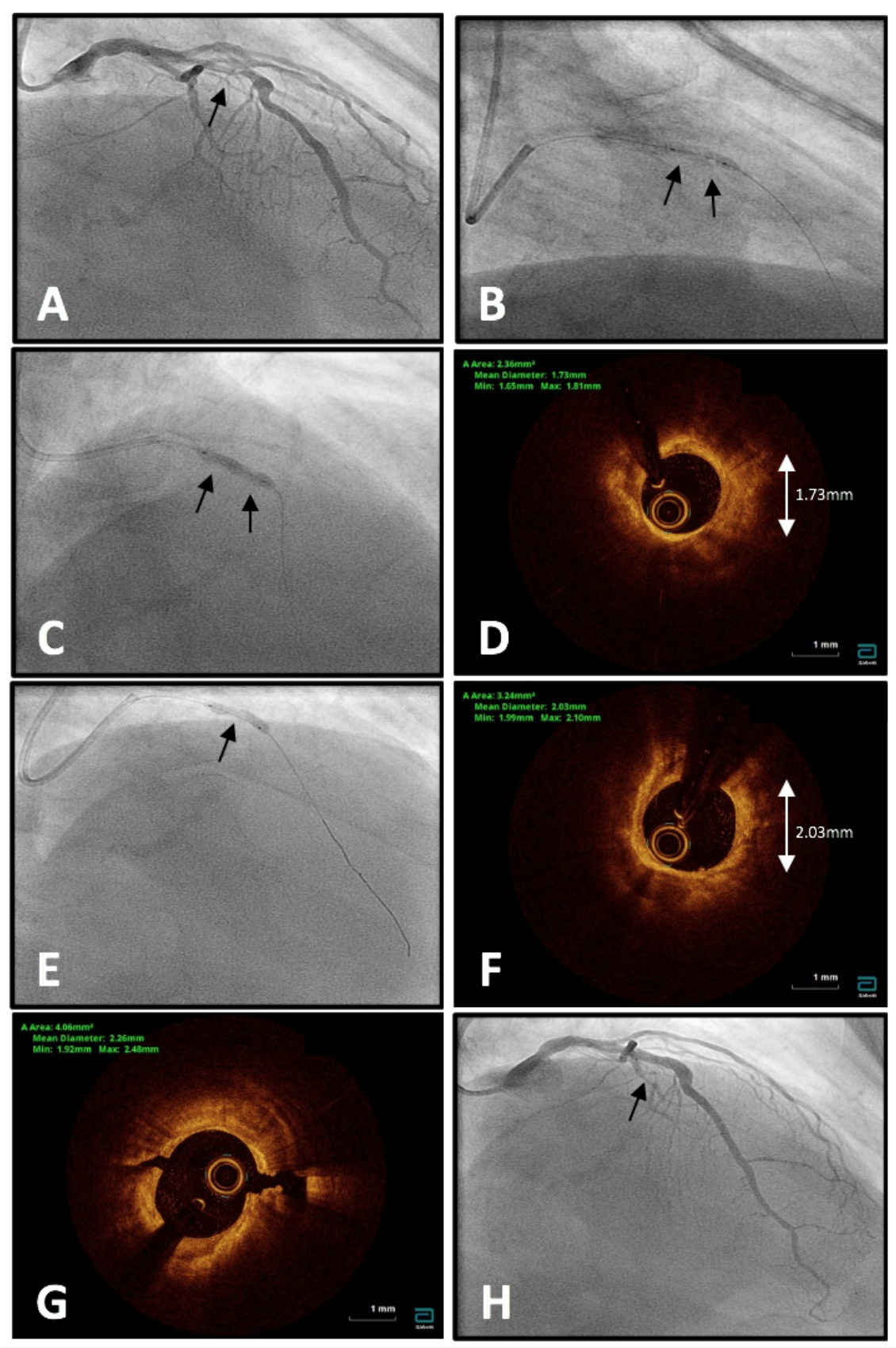



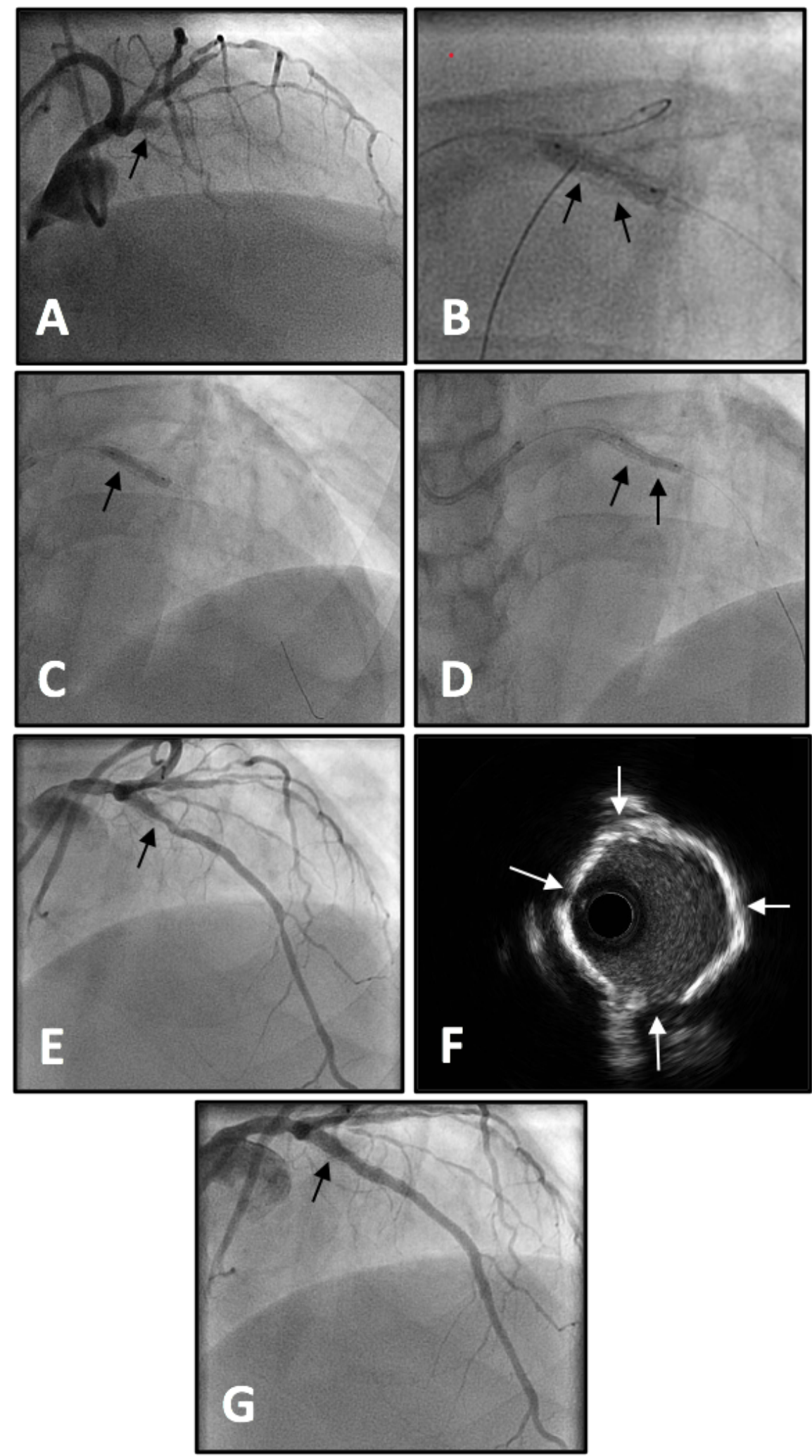